\title{
A praxeologia da regra de três algebrizada e a proposição cinco do Liber Quadratorum
}

The praxeology of the rule of three algebraized and proposition five of the liber quadratorum

Denivaldo Pantoja da Silva ${ }^{1}$ José dos Santos Guimarães Filho² João Claudio Brandemberg ${ }^{3}$

\section{Resumo}

Este artigo tem como objetivo buscar uma transposição didática para o ensino da Regra de Três, uma prática sócio-histórica, que permita desenvolver sua algebrização em uma Praxeologia denominada Regra de Três Algebrizada como um processo evolutivo. Para isso, buscamos na História da Matemática, consultando obras da Matemática consideradas fontes, uma abordagem metodológica que aliada às noções de Transposição Didática, Praxeologia e Modelo Epistemológico de Referência como provedores do engendramento de um conjunto de regras, relações e procedimentos necessários para tal desenvolvimento. Nossos resultados iniciais apontam a proposição cinco do Liber Quadratorum como um dispositivo didático eficaz para iniciação ao estudo de práticas de algebrização, além de propor um fazer de modelização matemática possível na Educação Básica.

Palavras-chave: história da matemática; práxis; algebrização; regra de três; Liber Quadratorum.

\section{Abstract}

This article has as objective seek a didactic transposition for the teaching of the Rule of Three, a socio-historical practice, which allows to develop your algebraization in a Praxeology called Rule of Three Algebraized as an evolutionary process. For that, we searched in the History of Mathematics, consulting works of Mathematics considered sources, a methodological approach that combined with the notions of Didactic Transposition, Praxeology and Epistemological Model of Reference as providers of the engendering of a set of rules, relations and procedures necessary for such development. Our initial results point to the proposition five of the Liber Quadratorum as an effective didactic device to initiate the study of practices of algebraization, in addition to proposing a make possible of mathematical modeling in Basic Education.

Keywords: history of mathematics; praxis; algebrization; rule of three; Liber Quadratorum.

\footnotetext{
${ }^{1}$ Universidade Federal do Pará | denivaldo@ufpa.br

2 Universidade Federal do Pará | jsguimaraes@ufpa.br

${ }^{3}$ Universidade Federal do Pará | brand@ufpa.br
} 


\section{Introdução}

O ensino da Regra de Três, uma prática sócio-histórica, tem ocupado espaço em pesquisas na historiografia da Matemática (SMITH,1958; SARMA, 2002; HØYRUP,2007) e em Didática das Matemáticas (BOSCH,1994; COMIN,2000; GARCIA,2005) nesse caso, tomando como base a Teoria Antropológica do Didático (TAD, daqui em diante). Em geral, essas investigações buscam respostas possíveis para determinadas problemáticas com foco nas aprendizagens e nas organizações praxeológicas, propõem métodos baseados em ações didáticas em sala de aula, que contemplam, a concepção, realização, observação e análise de sequências de ensino, permitindo validação interna a partir da confrontação das análises a priori e a posteriori, nesse caso, trata-se da engenharia didática (ARTIGUE, 1988).

A Regra de Três traz consigo um percurso histórico e evolutivo que integra dimensões do tipo epistemológica, didática e sobretudo prática determinando, entre outros aspectos, maiores possibilidades de investigação. Nessa linha, sua historicidade impressa no tempo pode proporcionar o instrumental necessário para compreender as atividades que as escolas desenvolvem atualmente, de tal modo a evidenciar o papel construtivo e formativo que joga o processo de ensino e aprendizagem da Matemática.

Desse modo, não há dúvidas que a História da Matemática como um campo de estudo e investigação científica disponha em seu escopo objetos - textos e obras - com potencialidades didáticas que proporcionam ao professor a oportunidade de desenvolver práticas de ensino diferenciadas para o trabalho em sala de aula de forma contextualizada, interdisciplinar e com significados. Para Roque e Pitombeira (2012),

A Matemática pode ser ensinada de uma maneira mais "concreta", caso seus conceitos forem tratados a partir de um contexto. Isto não significa necessariamente partir de um problema quotidiano, e sim saber com o que estes conceitos se relacionam, como podem ser inseridos em uma rede de relações e de significados - ainda que estas relações pertençam à própria Matemática (ROQUE e PITOMBEIRA,2012, p.vii).

Neste sentido, recorremos à historiografia da Matemática, em particular, ao texto da proposição cinco do Liber Quadratorum, obra atribuída ao matemático Fibonacci como fonte histórica provedora relativamente completa que dispõe de um conjunto de regras, relações e procedimentos operatórios fundamentais para desenvolver o estudo da Regra de Três e alcançar a forma algebrizada.

Fibonacci, alcunha dada por Guillaume Libre a Leonardo de Pisa (1180 - 1250), por volta de seiscentos anos após sua morte; sendo outorgado a ele o título de primeiro matemático medieval por Castillo (2007). Pode ser observado ao longo da história que este proeminente matemático, deixou contribuições reconhecidas até hoje, entre elas a obra denominada Liber Quadratorum - o Livro dos Quadrados.

A Europa no período medieval, fazia uso de uma estabilidade, pois já não haviam tantas guerras, permitindo a expansão agrícola e demográfica, o que permitiu a Fibonacci estudar de forma mais concisa, viajando pelo norte da África e conhecendo novas matemáticas. Neste momento, entra em cena um rei intelectual, o qual, apreciava a arte e a ciência, a pedido dele - Frederico II - foi organizado um torneio matemático, que teve como um de seus convidados especiais, Fibonacci, que ganha admiração do rei após a repercussão de seu primeiro livro intitulado o Liber Abaci (1202). 
Neste torneio são propostos três problemas matemáticos, sendo todos solucionados por Fibonacci (CASTILLO, 2007).

Dentre os problemas propostos a Fibonacci, damos ao primeiro uma atenção especial. O problema consiste em "encontrar um número quadrado que adicionado ou subtraído cinco permaneça um número quadrado", o qual, motivou a construção do Liber Quadratorum. Outro ponto a ser evidenciado é que este livro foi dedicado ao rei Frederico II, escrito em 1225 (SIGLER, 1987).

Fibonacci para escrever o livro, parte da ideia que consiste no fato de a soma de números ímpares consecutivos ter uma relação direta com os números quadrados, sendo apresentado na introdução do livro e demonstrado na quarta proposição deste (GUIMARÃES FILHO, 2018).

O Liber Quadratorum é composto de vinte e quatro problemas, apresenta, segundo Oliveira (2013), em seu conteúdo, problemas envolvendo a Teoria dos Números que, dentre outros, examina métodos para encontrar as ternas pitagóricas de várias formas.

Podemos assim, objetivar, neste artigo, a busca por uma transposição didática, no sentido do "trabalho que transforma um objeto de um saber a ensinar a um objeto de ensino" (CHEVALLARD,1991, p.45), para a Regra de Três que permita desenvolver a algebrização da prática, o que determina uma praxeologia denominada Regra de Três Algebrizada, tomando a proposição cinco do Liber Quadratorum - "Encontre dois números quaisquer de modo que a soma de seus quadrados forme um quadrado, e que este quadrado possa ser formado pela soma de dois quadrados quaisquer diferentes dos dois primeiros" - como Modelo Epistemológico de Referência (MER).

Vamos considerar que um MER é um instrumento teórico que nos permite entre outras funções analisar, reconstruir organizações praxeológicas, por em relevo com outros modelos existentes - contraste - (SIERRA, 2007), investigar e até mesmo engendrar regras e procedimentos para o estudo de um fenômeno didático. Mas, centraremos nossa atenção na análise, desenvolvimento e (re) construção de Praxeologias, palavra que resume a ideia de que "toda atividade humana regularmente realizada pode ser descrita com um modelo único, a praxeologia" (CHEVALLARD, 1999, p.222) de certo modo já naturalizadas e, com isso, incentivar a reflexão sobre práticas docente no ensino da Matemática que evoquem o caráter investigativo.

Assim, temos a convicção de que para promover aprendizagem matemática com significados para o aluno e para o professor, uma estratégia razoável pode se dar pela reconstrução histórica do objeto matemático em termos de praxeologias ou organizações praxeológicas que de acordo com Chevallard (1999) está constituída por um bloco práticotécnico $[\mathrm{T} / \mathrm{O}]$ e outro tecnológico-teórico $[\Theta / \odot]$. "O bloco $[\Theta / \odot]$ se identifica habitualmente como um saber, enquanto que o bloco [T/Ô] constitui um saber-fazer. Por metonímia se designa correntemente como "saber" a praxeologia $[T / \hat{O} / \Theta / \odot]$ completa, ou inclusive qualquer parte dela" (CHEVALLARD,1999, p.226).

A (re) construção de uma praxeologia por meio da transposição didática, pode se constituir em um contexto próprio de aprendizagem, para discutir, desenvolver e investigar conjuntamente alunos e professores as sutilezas interpostas no processo de ensino, permitirá compreender o desenvolvimento histórico-epistemológico de noções matemáticas estudadas, evitando, por exemplo, o que Schubring (2018) alerta sobre o uso da História da Matemática por interpretações ingênuas. 
A proposição cinco como MER assumido no sentido posto, pode favorecer o processo de construção do conhecimento matemático, ou seja, proporcionar a iniciação ao estudo de problemas escolares, neste caso, de Regra de Três a avançar progressivamente até sua forma algebrizada. O fazer matemático mobilizado na demonstração realizada por Fibonacci, pela álgebra geométrica, poderá promover a iniciação dos alunos a estudos em níveis mais avançados da Matemática. Portanto, a referida proposição apresenta relações potencialmente favoráveis ao desenvolvimento de um dispositivo didático eficaz, tomado aqui como "tudo aquilo que está relacionado com o estudo e com a ajuda para o estudo da Matemática" (CHEVALLARD, BOSCH, GASCON, 2001, p.46).

Desse modo, propomos a seguinte questão como orientadora deste trabalho: Como desenvolver uma transposição didática, a partir da proposição cinco de Fibonacci para Regra de Três, na forma algebrizada, utilizando a álgebra elementar como instrumento de modelização matemática?

Para enfrentá-la, tomamos a proposição cinco demonstrada por Fibonacci como Modelo Epistemológico de Referência, partindo da hipótese que poderá dispor da infraestrutura necessária para o desenvolvimento do estudo da Regra de Três culminando com a algebrização, uma vez que a escola, em geral, aplica a Regra de Três na resolução de problemas de proporcionalidade geométrica.

A seguir, discutimos a proposição cinco do Liber Quadratorum como MER.

\section{A proposição cinco: modelo epistemológico de referência para regra de três algebrizada}

Como evidenciamos anteriormente, Fibonacci enuncia a proposição cinco do seguinte modo: Encontre dois números quaisquer de modo que a soma de seus quadrados forme um quadrado, e que este quadrado possa ser formado pela soma de dois quadrados quaisquer diferentes dos dois primeiros.

Para a demonstração dessa proposição, nos apropriamos da demonstração de Fibonacci com alguns ajustes na linguagem algébrica, com o intuito de deixar a compreensão mais acessível. Mesmo com alguns ajustes na linguagem algébrica, permanecemos com partes consideráveis da simbologia usada no século XIII por Fibonacci ${ }^{4}$.

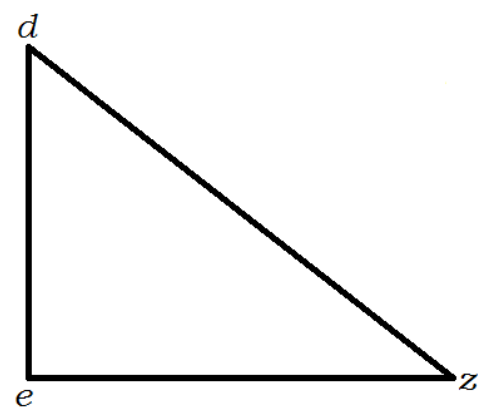

Figura 1 - Representação geométrica proposta por Fibonacci. Fonte:

Guimarães Filho (2018)

\footnotetext{
${ }^{4}$ Para maiores informações ou esclarecimentos consulte Guimaraes Filho (2018).
} 
Dessa forma, Fibonacci toma dois números, os quais, resultam em um quadrado com a soma de seus quadrados: $\boldsymbol{a}^{2}+\boldsymbol{b}^{2}=\boldsymbol{g}$. Da proposição, temos que encontrar outros dois números, os quais, somados resultem no número $\boldsymbol{g}$, o qual, é um número quadrado. Para esse momento Fibonacci usa um triângulo retângulo (Figura 1) como artifício para encontrar esses dois novos números, os quais, são representados pelos catetos desse triangulo retângulo, sendo a hipotenusa o novo quadrado formado.

Desta forma teremos duas situações, o quadrado pode ser igual ou diferente ao número $\boldsymbol{g}$. Se for igual admitimos que a hipotenusa será igual ao número $\boldsymbol{g}$. Se for diferente, teremos dois desdobramentos, a hipotenusa pode ser menor ou maior que o número quadrado $\boldsymbol{g}$.

Ao observar que a hipotenusa pode ser maior que o número $\boldsymbol{g}$, Fibonacci constrói um triangulo semelhante (Figura 2), dessa forma, determina-se uma constante de proporcionalidade $\boldsymbol{C}$, a partir das seguintes relações:

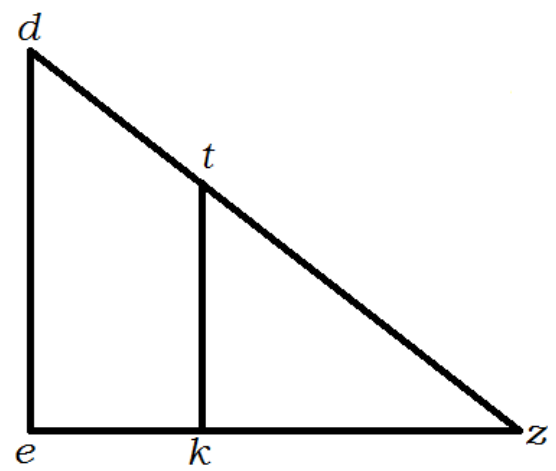

Figura 2 - Representação geométrica proposta por Fibonacci. Fonte:

Guimarães Filho (2018)

$$
\begin{gathered}
>\frac{\overline{t z}}{\overline{d z}}=C>\frac{\overline{t k}}{\overline{d e}}=C>\frac{\overline{k z}}{\overline{e z}}=C \\
>\overline{t k}^{2}+\overline{k z}^{2}=\overline{t z}^{2}=g
\end{gathered}
$$

Observamos da figura 2 que $\overline{\boldsymbol{t} \boldsymbol{k}}=\frac{\overline{\boldsymbol{z}} \boldsymbol{\overline { \boldsymbol { d } }}}{\overline{\boldsymbol{z}}} \overline{\boldsymbol{d e}} \Rightarrow \overline{\boldsymbol{t} \boldsymbol{k}}=\boldsymbol{C} * \overline{\boldsymbol{d e}}$, assim como, $\overline{\boldsymbol{k z}}=\frac{\overline{\boldsymbol{z}} \boldsymbol{\overline { \boldsymbol { t } }}}{\overline{\boldsymbol{z}}} * \overline{\boldsymbol{e z}} \Rightarrow$ $\overline{\boldsymbol{k}} \mathbf{Z}=\boldsymbol{C} * \overline{\boldsymbol{e Z}}$, desta forma encontramos dois valores proporcionais, os quais a soma de seus quadrados resultam em $\boldsymbol{g}$.

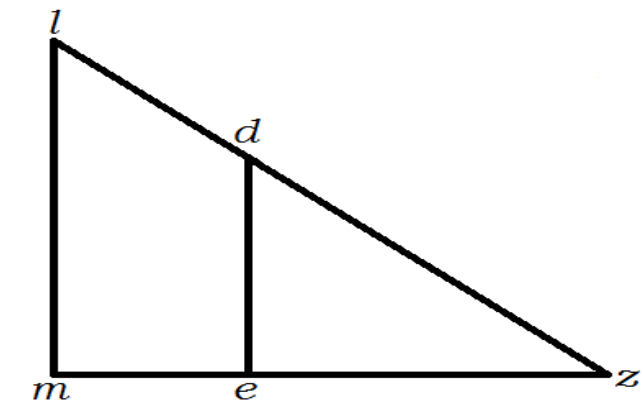

Figura 3 - Representação geométrica proposta por Fibonacci. Fonte: Guimarães Filho (2018) 
Dando seguimento na demonstração construída por Fibonacci, temos a hipotenusa menor que $\boldsymbol{g}$, para esta situação, de igual forma, é construído um triangulo semelhante (Figura 3) ao primeiro (Figura 1), assim, teremos as seguintes relações, onde denominamos $\boldsymbol{C}$ como uma constante de proporcionalidade.

$$
\begin{gathered}
>\frac{\overline{z l}}{\overline{d z}}=C>\frac{\overline{d e}}{\overline{l m}}=C>\frac{\overline{e z}}{\overline{m z}}=C \\
>\overline{l m}^{2}+\overline{m z}^{2}=\overline{l z}^{2}=g
\end{gathered}
$$

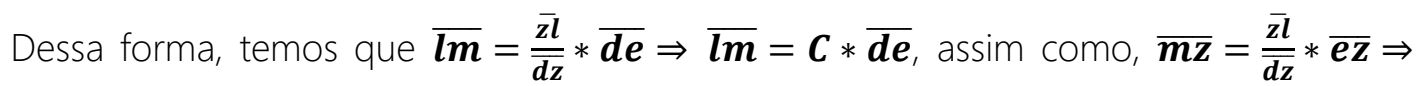
$\overline{\boldsymbol{k z}}=\boldsymbol{C} * \overline{\boldsymbol{e z}}$. Como na situação anterior, Fibonacci gera dois valores proporcionais, onde a soma de seus quadrados gera o número quadrado $\boldsymbol{g}$. Desta forma, podemos afirmar que Fibonacci consegue encontrar dois números quaisquer de modo que a soma de seus quadrados forme um quadrado, e que este quadrado possa ser formado pela soma de dois quadrados quaisquer diferentes dos dois primeiros. É importante evidenciar que em todos os modelos propostos para a constante de proporcionalidade $\boldsymbol{C}$, Fibonacci opta pela relação da menor medida pela maior, exceto em $\frac{\overline{\mathbf{z}}}{\overline{\mathbf{d} \boldsymbol{z}}}$, portanto, somente relacionando a maior medida pela menor, isto é, $\overline{\overline{\boldsymbol{z}} \mathbf{l}}$, levará a resposta correta, fato esse que Fibonacci, em seu tempo, provavelmente identificou.

A fim de elucidar a demonstração de Fibonacci, apresentamos um exemplo encontrado em seu livro para essa proposição, apresentado da seguinte forma.

Para:

$$
a=5>b=12>g=169
$$

Temos que,

$$
a^{2}+b^{2}=g \Rightarrow 5^{2}+12^{2}=169 \Rightarrow 25+144=169=13^{2}
$$

E se temos $\overline{\boldsymbol{d}}^{2}>\boldsymbol{g}$ e admitindo,

$$
>\overline{d e}=15>\overline{e z}=8>\overline{d z}=17>\overline{t z}=13
$$

Temos que,

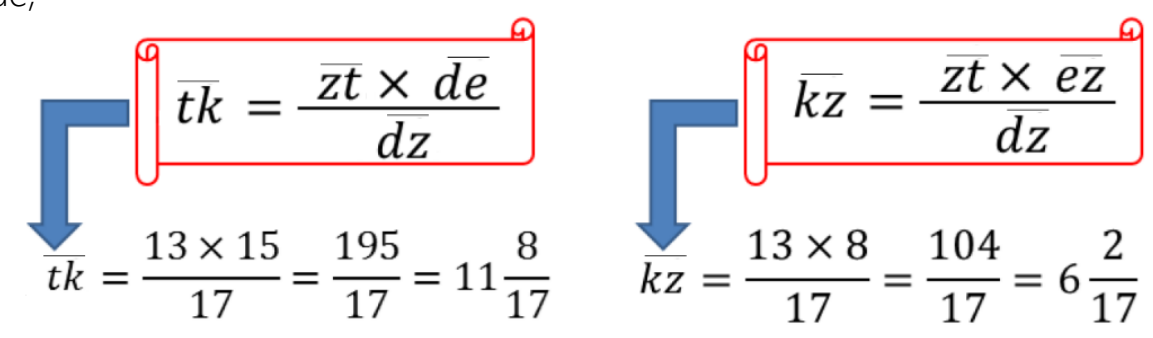

Dessa forma, podemos apresentar o desenvolvimento seguinte para provar a conjectura de Fibonacci para a situação de $\overline{\boldsymbol{d z}}^{2}>\boldsymbol{g}$, assim,

$$
\overline{t k}^{2}+\overline{k z}^{2}=\overline{t z}^{2} \Rightarrow\left(\frac{195}{17}\right)^{2}+\left(\frac{104}{17}\right)^{2}=13^{2} \Rightarrow \frac{38025}{289}+\frac{10816}{289}=13^{2}
$$




$$
\Rightarrow \frac{48841}{289}=169=13^{2}=g
$$

E se temos $\overline{\boldsymbol{d z}}^{2}<\boldsymbol{g}$ e admitindo que,

Temos que,

$$
>d e=4>e z=3>d z=5>l z=13
$$

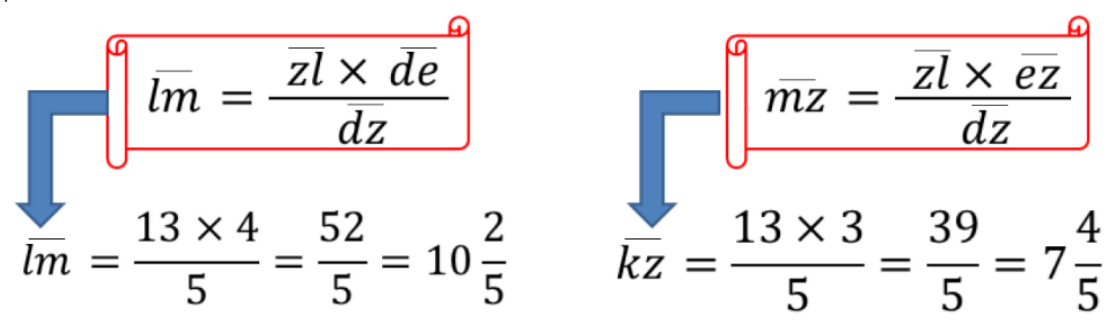

Como no caso anterior, segue o desenvolvimento para provar a conjectura de Fibonacci para a situação em que $\overline{\boldsymbol{d z}}^{2}<\boldsymbol{g}$, assim,

$$
\begin{aligned}
\overline{l m}^{2}+\overline{m z}^{2}=\overline{l z}^{2} & \Rightarrow\left(\frac{52}{5}\right)^{2}+\left(\frac{39}{5}\right)^{2}=13^{2} \Rightarrow \frac{2704}{25}+\frac{1521}{25}=13^{2} \\
& \Rightarrow \frac{4225}{25}=169=13^{2}=. g .
\end{aligned}
$$

Logo,

Para $\boldsymbol{d z ^ { 2 }}=\boldsymbol{g}$, temos $\boldsymbol{a}^{2}+\boldsymbol{b}^{2}=\boldsymbol{g}=\overline{\boldsymbol{d e}}^{2}+\overline{\boldsymbol{e z}}^{2}$

$>$ Para $\overline{\boldsymbol{d z}}^{2}>\boldsymbol{g}$, temos $\boldsymbol{a}^{2}+\boldsymbol{b}^{2}=\boldsymbol{g}=\overline{\boldsymbol{t}}^{2}+\overline{\boldsymbol{k z}}^{2}$;

$>$ Para $\overline{\boldsymbol{d z}}^{2}<\boldsymbol{g}$, temos $\boldsymbol{a}^{2}+\boldsymbol{b}^{2}=\boldsymbol{g}=\overline{\boldsymbol{l m}}^{2}+\overline{\boldsymbol{m z}}^{2}$.

Com isso, Fibonacci demonstra como podem ser encontrados infinitos valores para esta proposição, bem como, foi possível perceber que basta encontrar uma constante de proporcionalidade e multiplicá-la por qualquer valor para determinar valores outros que obedeçam à relação expressa nesta proposição.

Percebemos que o texto de Fibonacci como Modelo Epistemológico de Referência, ainda que tomado somente o desenvolvimento da proposição cinco, dotada de um conjunto de procedimentos, relações, representações e regras operatórias, engendra uma organização praxeológica - praxeologia - em um contexto próprio de aprendizagem possível de ser executado pelo professor em sala de aula. Nesse sentido, acreditamos que o fenômeno da transposição didática pode encaminhar uma proposta de ensino da Regra de Três na forma algebrizada ou Regra de Três Algebrizada, promovendo, com isso, de forma eficiente a transição desse contexto histórico para outro momento didático com características e representações simbólicas de práticas matemáticas atuais, é o que pretendemos abordar a seguir.

\section{Desenvolvimento da regra de três algebrizada}

Para o desenvolvimento de nossa proposta vamos assumir a proposição cinco ajustada nos moldes dos problemas protótipos - problemas ditos/reconhecidos como de regra de três - estudados na escola: Se conhecidos dois números quadrados cuja soma resulta em 
um quadrado, quais outros dois números quaisquer diferentes dos primeiros que também formam o mesmo quadrado?

O problema pode ser resolvido pelo menos de dois modos: Primeiro, utilizaremos o método aritmético. $\bigcirc$ enunciado do problema permite escolher dois números: o primeiro 5 e o segundo 12. A soma de seus quadrados é 169 , isto é, $\mathbf{5}^{2}+\mathbf{1 2}^{\mathbf{2}}=\mathbf{1 6 9}$. Esse resultado corresponde a $13^{2}$ como era de se esperar! Mas, para os números desconhecidos, sua escolha dependerá de duas situações: a primeira, se os números tomados formarem o mesmo número quadrado e a segunda, os números formarem um número quadrado diferente. No primeiro caso, é trivial, não há o que fazer, pois atende plenamente as condições do problema. No segundo caso, temos que o número quadrado é diferente, logo, duas possibilidades devem ser consideradas: (i) o número quadrado é maior que o número quadrado dado (169); (ii) o número quadrado é menor que o número quadrado dado (169). Desse modo, teremos:

(i): Tomando os números 8 e 15, teremos que $\mathbf{8}^{2}+\mathbf{1 5}^{2}=\mathbf{2 8 9}$, observe que $289>$ 169 e ambos são números quadrados. Esse número foi tomado como referência para o cálculo dos dois números quaisquer desconhecidos que formarão o mesmo número quadrado dado inicialmente (169). Para isso estabeleceremos relações adequadas de proporcionalidade do número 13 que corresponde a raiz quadrada de 169 a partir da relação com 17, raiz quadrada de 289, com o 8 para determinar o primeiro valor desconhecido. Em seguida repete-se o mesmo procedimento para o número 15. Dessa forma teremos as seguintes sentenças: 17 está para 8, assim como 13 está para o primeiro número desconhecido, que juntas formam a proporção. Do mesmo modo, 17 está para 15 assim como 13 está para o segundo número desconhecido. Da primeira sentença chegamos a $\frac{\mathbf{1 0 4}}{\mathbf{1 7}}$ e da segunda, $\frac{195}{\mathbf{1 7}}$, que são os dois números procurados.

Prova:

$$
\left(\frac{104}{17}\right)^{2}+\left(\frac{195}{17}\right)^{2}=\left(\frac{10816}{289}\right)+\left(\frac{38025}{289}\right)=\frac{48841}{289}=169=13^{2} .
$$

(ii): tomando os números 3 e 4 , teremos que $\mathbf{3}^{2}+\mathbf{4}^{\mathbf{2}}=\mathbf{2 5}$, observe que $25<169$ e ambos são números quadrados. Esse número foi tomado como referência para o cálculo dos dois números quaisquer desconhecidos que formarão o mesmo número quadrado dado inicialmente (169). Para isso estabeleceremos relações adequadas de proporcionalidade do número 13 que corresponde a raiz quadrada de 169 a partir da relação com 5, raiz quadrada de 25, com o 3 para determinar o primeiro valor desconhecido. Em seguida repete-se o mesmo procedimento para o número 4. Dessa forma teremos a proporção: 5 está para 3, assim como 13 está para o primeiro número desconhecido. Do mesmo modo, 5 está par 4 assim como 13 está o segundo número desconhecido. Da primeira sentença chegamos a $\frac{\mathbf{3 9}}{\mathbf{5}}$ e da segunda, $\frac{\mathbf{5 2}}{\mathbf{5}}$, que são os dois números procurados.

Prova:

$$
\left(\frac{39}{5}\right)^{2}+\left(\frac{52}{5}\right)^{2}=\left(\frac{1521}{25}\right)+\left(\frac{2704}{25}\right)=\frac{4225}{25}=169=13^{2} .
$$

Portanto, finalizamos a resolução do problema utilizando o discurso aritmético analítico. Essa resolução adaptada de Fibonacci que acabamos de apresentar, pode ser utilizada pelo professor nas aulas inicialmente sem dificuldades de ordem operatória, 
embora, exigindo do aluno certa competência de análise, que se adquire possivelmente nas experiências em estudos aritméticos.

A Segunda maneira, leva em consideração o fato de que o problema permite outro modo de pensar a resolução, então evocamos a Regra de Três como método de resolução de problemas de proporcionalidade do modo escolar; é nesse momento que a proposição cinco jogará o papel de dispositivo didático - um Modelo Epistemológico de Referência para iniciação ao estudo da regra, pois terá a vantagem de ser um método prático, rápido e seguro. Senão vejamos:

Para esta resolução, iniciamos recorrendo a um esquema gráfico para executarmos a disposição dos dados do problema escrevendo os valores das grandezas de mesma espécie na mesma coluna; há dois tipos utilizados rotineiramente na escola apresentados geralmente nos livros didáticos, um recorre a um quadro e o outro a setas mostrados a seguir:

\begin{tabular}{|c|c|}
\hline 17 & 8 \\
\hline 13 & $x$ \\
\hline
\end{tabular}

$$
\mid \begin{aligned}
& 17 \\
& 13
\end{aligned}
$$$$
8
$$

Daí, segue-se a proporção que após breve analise sobre a proporcionalidade estabelecida entre as grandezas, atribuindo-se $\boldsymbol{x}$ para o número desconhecido e, em seguida, determina-se esse valor pelo produto cruzado dos termos da proporção como segue:

$$
\frac{17}{13}=\frac{8}{x} \Rightarrow 17 \times x=13 \times 8 \Rightarrow x=\frac{104}{17}
$$

Do mesmo modo, chegaremos ao outro número desconhecido:

$$
\frac{17}{13}=\frac{15}{y} \Rightarrow 17 \times y=13 \times 15 \Rightarrow y=\frac{195}{17}
$$

Portanto, os números procurados são $\frac{\mathbf{1 0 4}}{\mathbf{1 7}}$ e $\frac{\mathbf{1 9 5}}{\mathbf{1 7}}$.

Como podemos notar, essa resolução breve, mobiliza objetos e procedimentos da álgebra elementar que podem ser tratados pelo professor nas aulas nos diferentes níveis de ensino sem apresentar dificuldades operacionais, do modo como a praxeologia da Regra de Três "vive" na escola, em geral, como método para resolver problemas protótipos de proporcionalidade.

Em continuidade, para alcançar a algebrização do método da Regra de Três, a Regra de Três Algebrizada, o professor poderá também apresentar/aplicar o método aritmético da redução à unidade; esse último que apresentamos pode vir depois da redução à unidade, pois utiliza recursos algébricos em sua resolução e finalmente, a generalização do método para esse tipo de problema. A sequência pode avançar em complexidade seguindo para os tipos de problemas: inversamente proporcional, regra de três composta e problemas de aplicação da Regra de Três.

Essa evolução do estudo deverá culminar com o processo de generalização da Regra de Três. Silva (2011) mostrou que a Regra de Três pode ser tomada como uma prática de modelização matemática possível na escola, ele apresenta a modelização matemática de diferentes métodos de resolução. Extraímos a forma canônica para apresentar a Regra de Três Algebrizada a partir da organização dos dados conforme anunciamos anteriormente, 
de acordo com enunciado do problema que resultará no modelo para determinação da solução aplicando os mesmos procedimentos e regras como segue:

$$
\begin{aligned}
& \begin{array}{lll}
\boldsymbol{n} & \cdots \ldots & \boldsymbol{p} \\
\downarrow & & \downarrow
\end{array} \\
& n_{1 \ldots \ldots \ldots \ldots \ldots} \\
& \frac{n}{n_{1}}=\frac{p}{p_{1}} \rightarrow p=\frac{p_{1}}{n_{1}} n
\end{aligned}
$$

Essa forma algebrizada da Regra de Três pode ser também explorada a partir da utilização do quadro que apresentamos anteriormente substituindo os números por letras. Essa estrutura algebrizada proporciona possibilidades de conjecturas do cálculo do termo desconhecido que pode ser qualquer um dos dados do problema e obter o modelo de cada um, neste caso, para $\boldsymbol{p}_{\mathbf{1}}, \boldsymbol{n} \boldsymbol{e} \boldsymbol{n}_{\mathbf{1}}$ e para outras composições da regra, a regra de três composta.

\begin{tabular}{|c|c|c|c|}
\hline$n$ & $p$ & $q$ & $\ldots$ \\
\hline$n_{1}$ & $p_{1}$ & $q_{1}$ & $\ldots$ \\
\hline
\end{tabular}

$$
\frac{n}{n_{1}}=\frac{p}{p_{1}} \times \frac{q}{q_{1}} \times
$$

É importante destacar que esse modelo expresso, como dissemos anteriormente, representa genericamente as possibilidades que podem ser engendradas no desenvolvimento da Regra de Três, necessariamente, cada modelo dependerá da situação em contexto concreto a qual exigirá para cada (re)arranjo a análise da relação de dependência entre as grandezas envolvidas se direta ou inversamente proporcionais.

\section{Considerações finais}

Vimos que, explorar potencialidades didáticas partindo da proposição cinco do Liber Quadratorum como MER para o desenvolvimento da Regra de Três em sua forma algebrizada que ousamos desenvolver, em certa medida foi discutido não com a expectativa da transposição de um saber definitiva ou única, mas sim como ponto inicial a possibilitar o vislumbre de trabalhos futuros de aprofundamento, para construção, análise e investigação de organizações praxeológicas de complexidade crescente além das que estão textualizadas nos manuais escolares.

E ainda, este estudo nos faz perceber que o resgate de obras ou fontes históricas como - Liber Quadratorum para fins didáticos, possibilita o desenvolvimento de novas alternativas em termos de praxeologias, para o ensino da Matemática. Identificamos potencialidades didáticas nesta obra do século XIII, além das já evidenciadas por Guimarães Filho e Brandemberg (2017) e Guimarães Filho (2018), em relação aos conteúdos das séries escolares e que transcendem a obra, tais como: explorar as ternas pitagóricas, elaborar atividades envolvendo quadrados e potências, examinar a evolução da linguagem algébrica entre outros envolvendo epistemologia e História da Matemática.

Especificamente, nosso foco é o fazer da proposição cinco, assumindo como Modelo Epistemológico de Referência suficientemente capaz de engendrar regras, relações e 
procedimentos matemáticos para tentar construir a praxeologia da Regra de Três Algebrizada como uma transposição didática para escola, mas, revelou-se também, como dispositivo didático de modelização matemática. Vimos que esse dispositivo se mostrou eficaz para inicialização do estudo da Álgebra Básica e avançar para níveis mais elevados, e ainda, numa versão ampla pode ser tomada para preparação ao estudo de campos avançados da Matemática como a teoria das Proporções, Álgebra Geométrica entre outras.

Em Silva e Guimarães Filho (2019) foi anunciada como continuidade dos estudos o tratamento da Regra de Três Algebrizada para gêneros de problemas de proporcionalidades que aqui confirmamos a possibilidade concreta de realizarmos parte do que foi proposto. Particularmente, Silva e Guimarães Filho (2020) questionam: É possível construir uma praxeologia da Regra de Três na forma algebrizada? Nossa resposta ainda que parcialmente, como observamos, evidencia a Regra de Três Algebrizada, como eficiente dispositivo de modelização matemática para uso na Educação Básica capaz de integrar, no desenvolvimento, as dimensões aritmética, algébrica e geométrica nas aulas de Matemática.

Portanto, uma resposta à questão inicial que apresentamos para orientar este trabalho - Como desenvolver uma transposição didática a partir da proposição cinco de Fibonacci para Regra de Três denominada forma algebrizada, utilizando a álgebra elementar como instrumento de modelização matemática? - pode ter sido descrita de forma construtiva no decorrer deste trabalho. Nossa preocupação foi em mostrar que é possível desenvolver na Escola Básica o estudo da Regra de Três por meio de uma transposição didática, a praxeologia da Regra de Três Algebrizada recorrendo à História da Matemática como método de investigação em obras matemáticas tidas como frontes, em particular, utilizando - Liber Quadratorum.

As noções teóricas de Praxeologia, Transposição Didática e Modelo Epistemológico de Referencia que evocamos, permitiram conjuntamente com o uso metodológico da História da Matemática, construir uma compreensão relativamente de complexidade crescente sobre o desenvolvimento da Regra de Três até a forma algebrizada, um fazer matemático possível na Educação Básica.

Enfim, esperamos contribuir para o trabalho docente, promovendo uma reflexão sobre as práticas de ensino em Matemática desenvolvidas na escola, e que inspire trabalhos futuros para aprofundamentos, no sentido de construir novas compreensões sobre outros objetos matemáticos de ensino que possam ajudar o professor na construção e reconstrução de organizações didático-matemáticas que evidencie o fazer matemático escolar em termos de praxeologias que proporcione, para além de aprendizagens duradouras, novas investigações no campo da Educação Matemática.

\section{Referências bibliográficas}

ARTIGUE, Michèle. Ingénierie didactique. Recherches en didactique des mathématiques, v. 9, n. 3, p. 281-308, 1988.

BOSCH, Marianna. La dimensión ostensiva en la actividad matemática: El caso de la proporcionalidad. Tesis doctoral, UAB, Barcelona, 1994.

CASTILLO, Ricardo Moreno. Fibonacci: El Primer Matemático Medieval. 2a. ed. Coleção - La matemática em sus personajes. España: Nivola, 2007. 
CHEVALLARD, Yves. El análisis de las prácticas docentes en la teoría antropológica de lo didáctico. Recherches en Didactique des Mathématiques, v. 19, n. 2, p. 221-266, 1999.

CHEVALLARD, Yves. La transposición didáctica. Del saber sabio al saber enseñado, 1991.

CHEVALLARD, Yves. BOSCH, Marianna. \& GASCÓN, Josep. Estudar matemáticas: o elo perdido entre o ensino e a aprendizagem. Trad. Daisy Vaz de Moraes. Porto Alegre: Artmed, 2001.

COMIN, Eugène. Proportionnalité et fonction linéaire Caractères, causes et effets didactiques des évolutions et des réformes dans la scolarité obligatoire. Tese de Doutorado, Université Sciences et Technologies - Bordeaux I, 2000.

FIBONACCI. Liber Quadratorum. Pisa, 1225.

GARCÍA, F. J. La modelización como herramienta de articulación de la matemática escolar: de la proporcionalidad a las relaciones funcionales. Doctoral dissertation. Universidad de Jaén. 2005.

GUMARAES FILHO, José dos Santos; BRANDEMBERG, João Cláudio. Um estudo do Liber Quadratorum (1225) e suas potencialidades para o ensino de Matemática. REMATEC, n. 26, p. 71-85, 2017.

GUIMARÃES FILHO, José dos Santos. Um estudo do Liber Quadratorum (1225) de Leonardo Fibonacci (1180 - 1250) e suas Potencialidades para o Ensino de Matemática. Dissertação de Mestrado. Universidade Federal do Pará. Belém-PA, 2018.

HØYRUP, Jens. Further questions to the historiography of Arabic (but not only Arabic) mathematics from the perspective of Romance abbacus mathematics. In $9^{a}$ Colloque Maghrébins l' Histoire des Mathématiques Arabes. Tipaza, 12 a 14 dez, 2007.

OLIVEIRA, José Jackson de. Sequências de Fibonacci: possibilidades de aplicações no ensino básico. Salvador: Edufba, 2013.

PAIS, Luiz Carlos. Ensinar e aprender matemática. Autêntica, 2006.

ROQUE, Tatiana; CARVALHO, João Bosco Pitombeira de. Tópicos de história da matemática, 2012 (Coleção ProfMat).

SARMA, Sreeramula Rajeswara. Rule of Three and its Variations in India. In: From China to Paris: 2000 Years Transmission of Mathematical Ideas, p. 133-156, 2002.

SCHUBRING, Gert. Os números negativos: exemplos de obstáculos epistemológicos? Editora livraria da física, São Paulo, 2018.

SIERRA,Tomaz Angel. Lo Matemático en el Diseño y Analisis de Organizaciones Didácticas: los sistemas de numeración y la medida de magnitudes. Tese de Doutorado. Universidad Complutense de Madrid,2007. Disponível em: https://eprints.ucm.es/7373/, acesso em 27/12/2019.

SIGLER, Laurence Edward. The Book of Squares. An annotated traslation into modern english. Academic Press, USA: 1987.

SILVA, Denivaldo Pantoja da. Regra de três: prática escolar de modelagem matemática. 2011.Dissertação (Mestrado em Educação em Ciências e Matemáticas), Instituto de Educação Matemática e Científica, Universidade Federal do Pará, Belém-PA. 
SILVA, Denivaldo Pantoja da. A invariável prática da regra de três na escola. 2017. Tese. (Doutorado em Educação em Ciências e Matemáticas), Instituto de Educação Matemática e Científica, Universidade Federal do Pará, Belém-PA.

SILVA, Denivaldo Pantoja da; GUIMARÃES FILHO, José dos Santos. Proposição cinco do Liber Quadratorum: a regra de três como dispositivo didático para proporcionalidade. Anais XIII Seminário Nacional de História da Matemática, 2019.

SMITH, David Eugene. History of mathematics. Courier Corporation, 1958. 